Supporting information

for

\title{
Quadruple Hydrogen Bonding Supramolecular Elastomers for Melt Extrusion Additive Manufacturing
}

Xi Chen, ${ }^{1}$ Callie E. Zawaski, ${ }^{2}$ Glenn A. Spiering, ${ }^{1}$ Boer Liu, ${ }^{1}$ Christina M. Orsino, ${ }^{1}$ Robert B. Moore, ${ }^{1}$ Christopher B. Williams, ${ }^{2}$ and Timothy E. Long ${ }^{1, *}$

${ }^{1}$ Department of Chemistry, Macromolecules Innovation Institute (MII), Virginia Tech, Blacksburg, VA, 24061

${ }^{2}$ Department of Mechanical Engineering, Macromolecules Innovation Institute (MII), Virginia Tech, Blacksburg, VA, 24061

*Email: telong@vt.edu

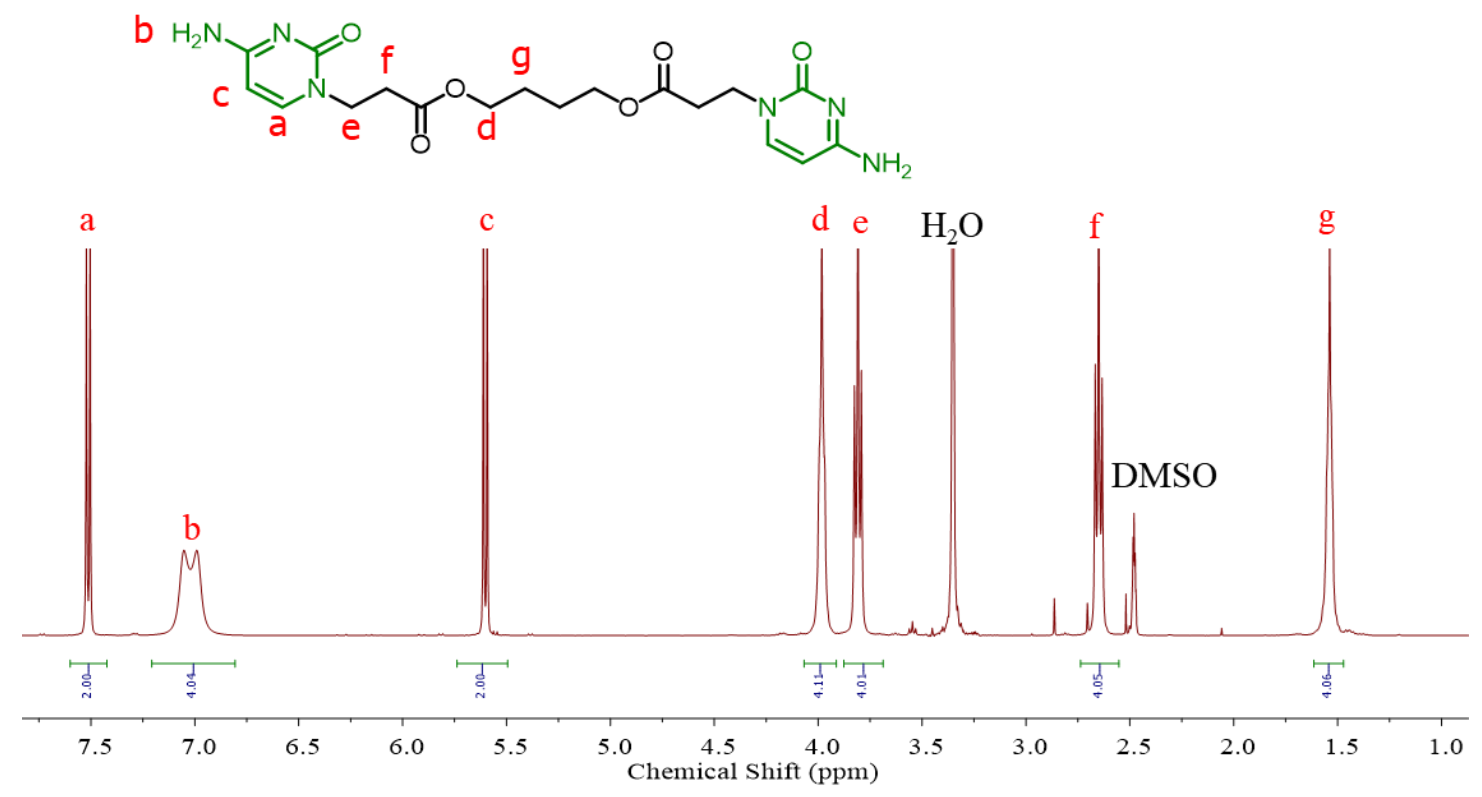




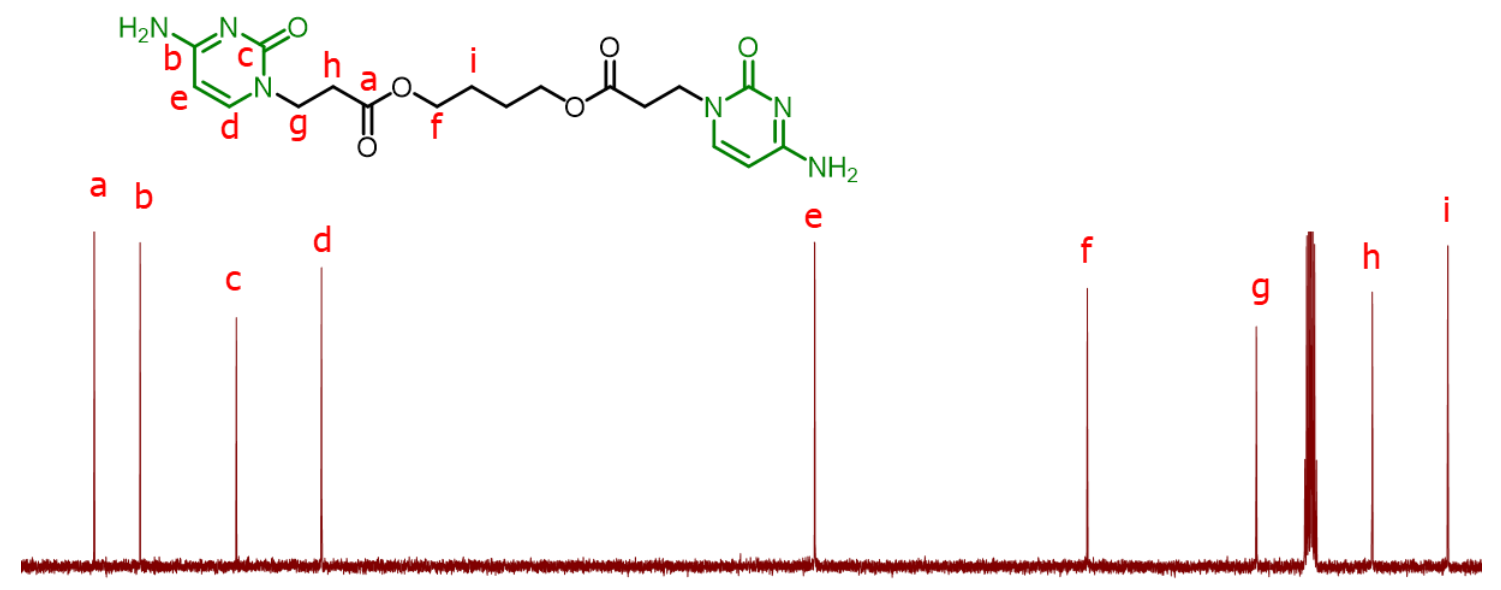

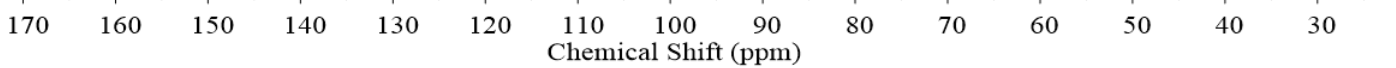

Figure S1. ${ }^{1} \mathrm{H}$ and ${ }^{13} \mathrm{C}$ NMR spectra of bis-cytosine monomer in DMSO- $d_{6}$.

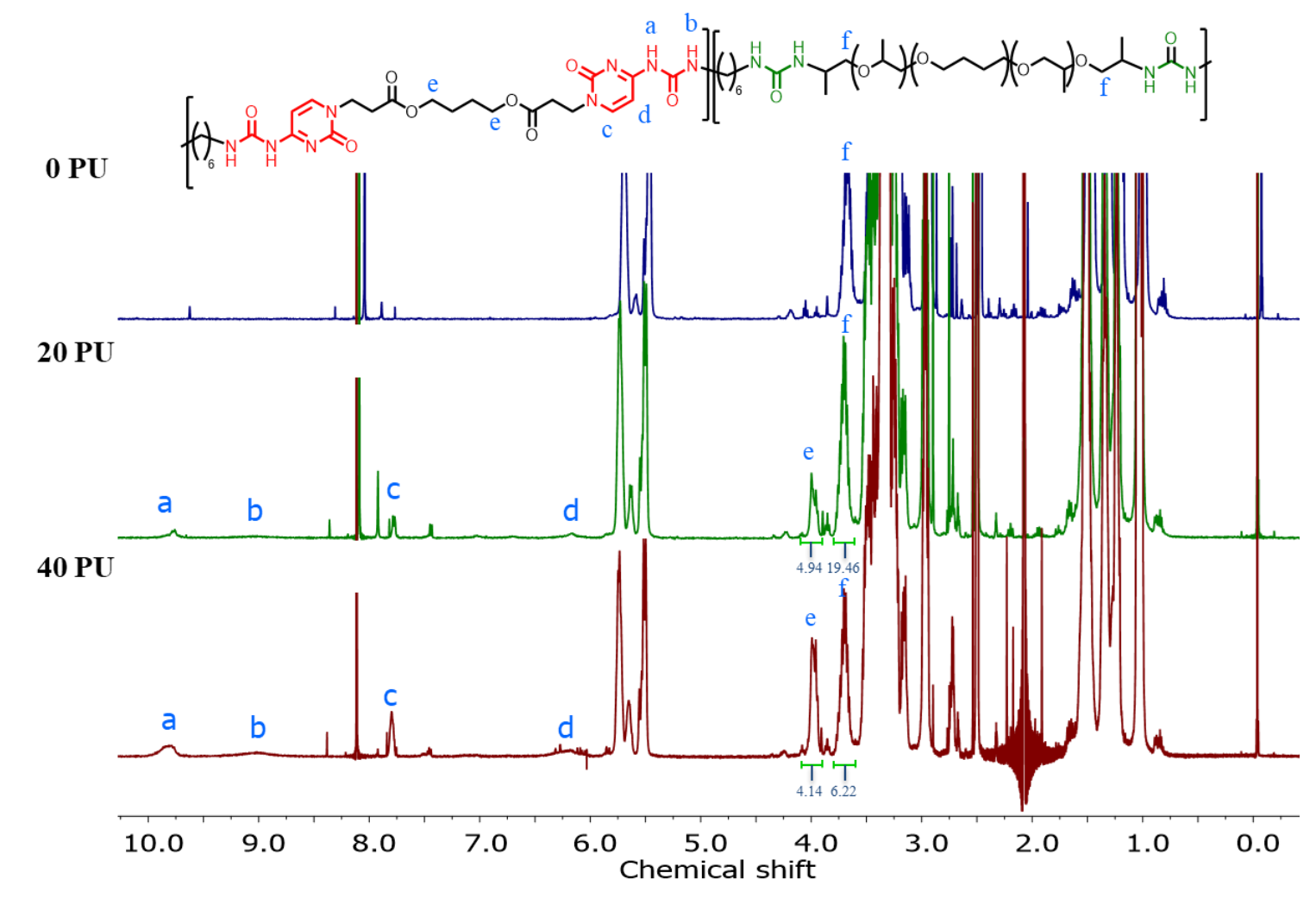

Figure S2. ${ }^{1} \mathrm{H}$ NMR spectra of 0-40 PU in DMSO- $d_{6}+\mathrm{CDCl}_{3}$. The ratio between e and $\mathrm{f}$ was used to calculate the hard segment content. 


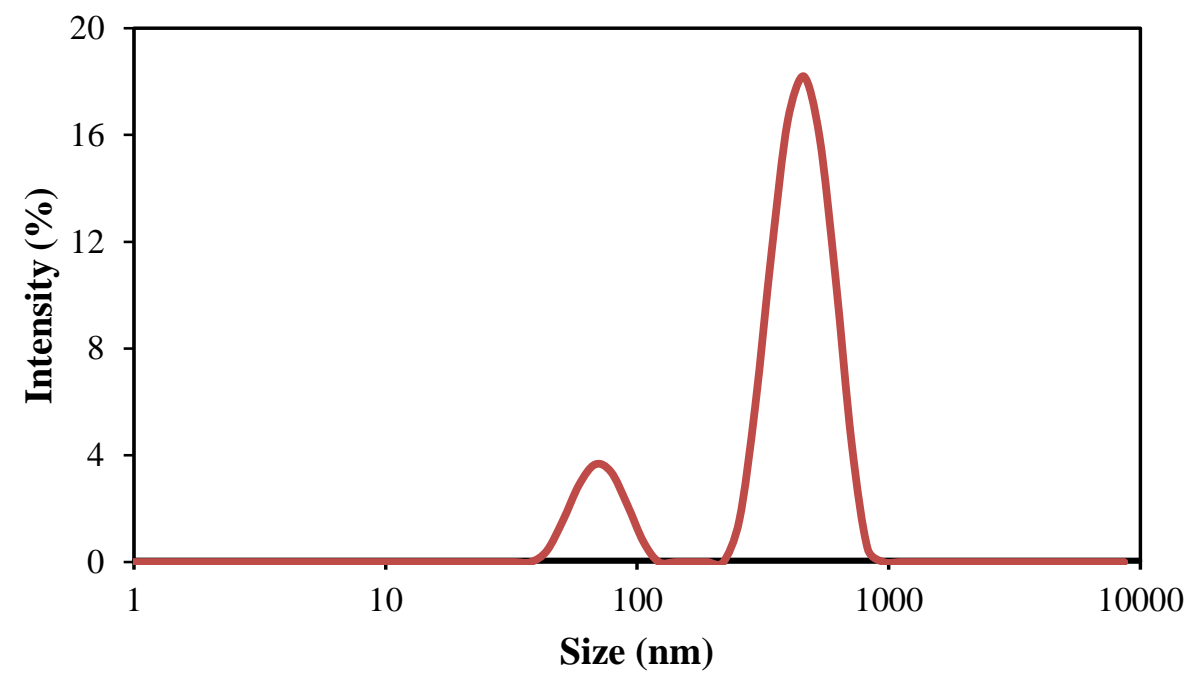

Figure S3. Size distribution of 40 PU measured by DLS in DMF.

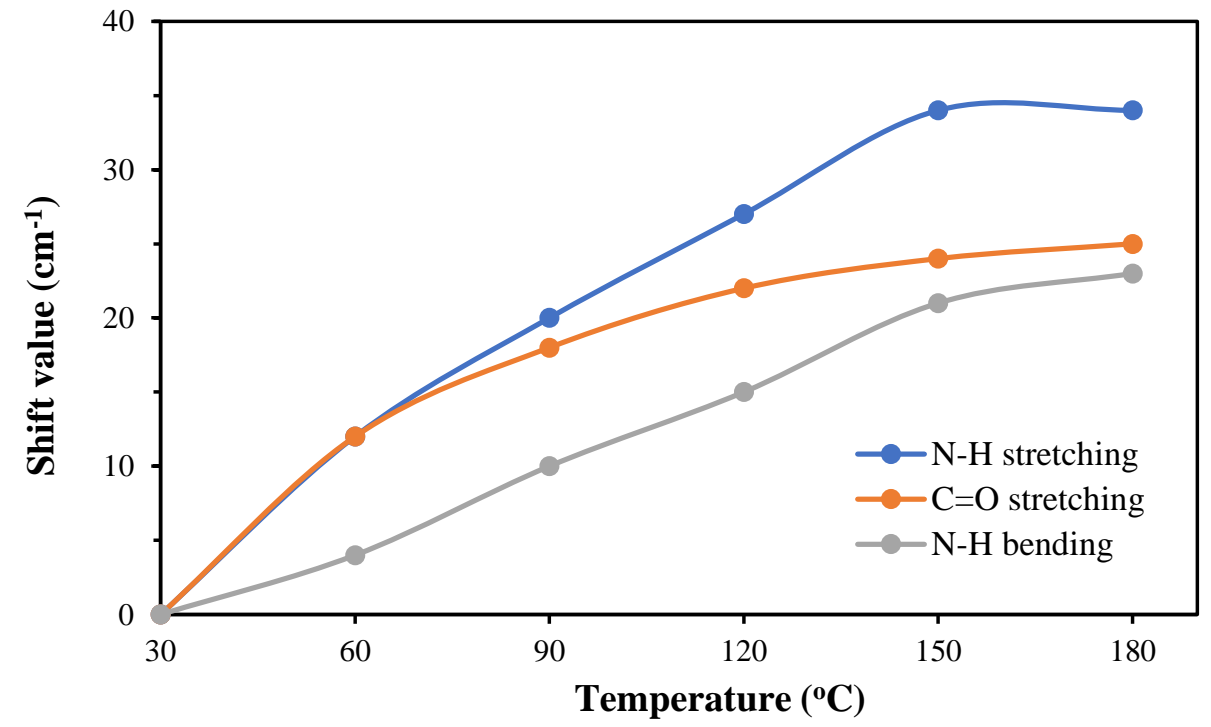

Figure S4. VT-FTIR spectral shifting values of $\mathrm{N}-\mathrm{H}$ stretching, $\mathrm{C}=\mathrm{O}$ stretching, and $\mathrm{N}-\mathrm{H}$ bending vibration as a function of temperature. 


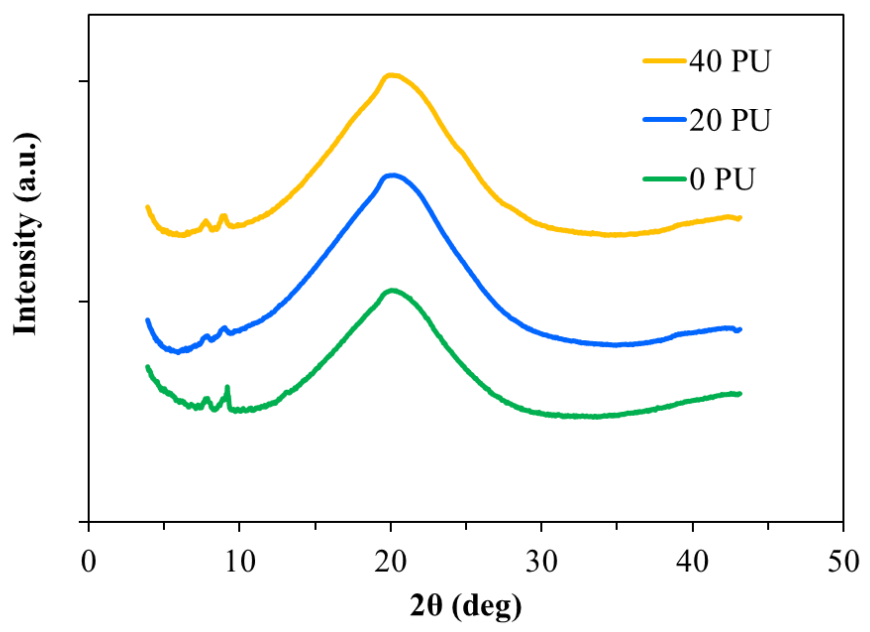

Figure S5. WAXS of the polyurea samples, the small sharp peaks located at 7-9 deg indicate the crystalline regions within the polymer.

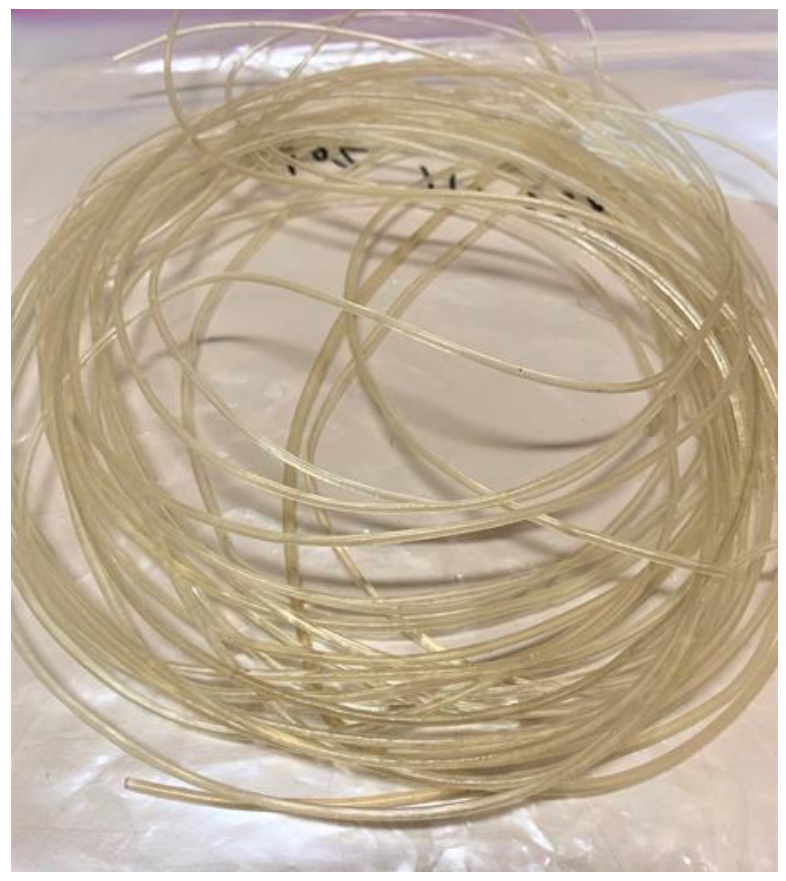

Figure S6. Extruded 20 PU filaments for FDM. 

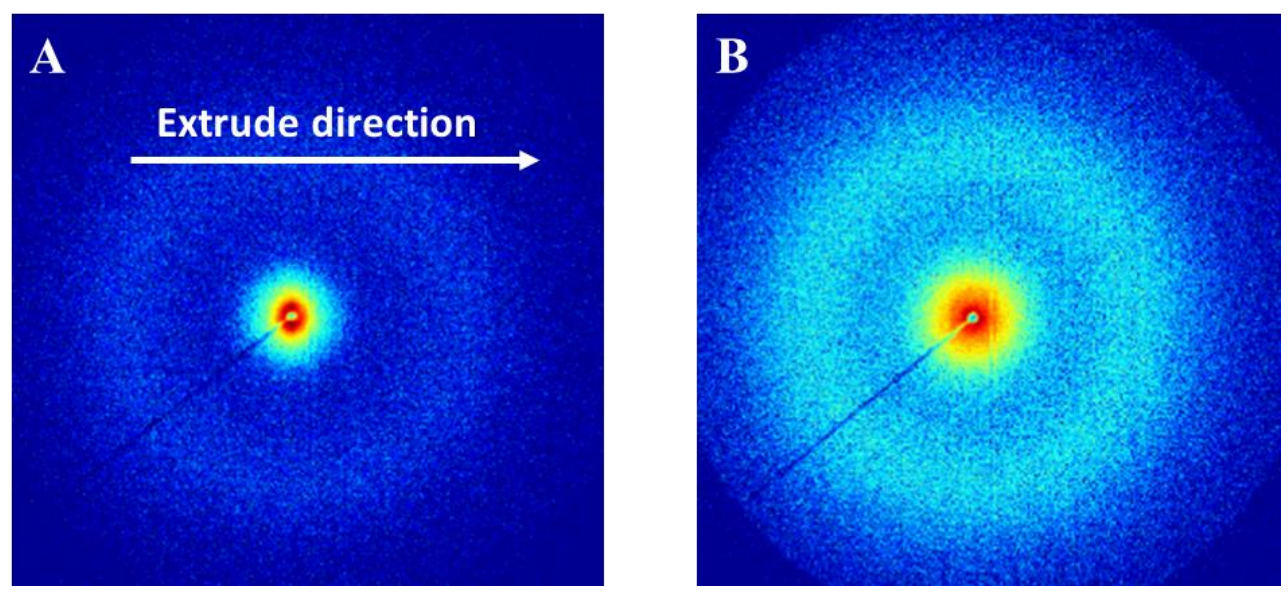

Figure S7. 2D SAXS patterns of (A) extruded 20 PU filament and (B) compression-molded 20 PU film. 